\title{
Method for the Estimation of the Limit Coefficients of Reflection and Transition of a Flat Micro Wave in a Bi-complex Medium
}

\author{
Anitm Yordanov ${ }^{1}$ \\ 1 - Technical University of Varna, Department of Electronics and Microelectronics, 9010, 1 Studentska Street, Varna, Bulgaria \\ Corresponding author contact: anyordegmail.com
}

\begin{abstract}
The reaction of different materials to external magnetic fields depends on the frequency of propagation of radio waves in substances and is determined by the dielectric constant $\hat{\varepsilon}$, magnetic permeability $\hat{\mu}$, by the vectors of the electric and magnetic field. When irradiated with a flat wave on a flat surface, the phenomenon occurs at the boundary between two media, which will be described by an algorithm through reflection and transmission coefficients used in the processing of transmitted and reflected signals from radars, and adopted in radiology as well.
\end{abstract}

Keywords: algorithm, microwave, permeability, reflection

\section{$1 \quad$ Introduction}

The polarization of materials caused by microwave fields does not change instantly, so the dielectric and magnetic permeability are presented as complex functions of the angular frequency $\boldsymbol{\omega}$. To compile a simple algorithm for establishing the reflection and transmission coefficients of ultrahigh frequency electromagnetic waves in metals and low-loss dielectrics, it shall be assumed that the considered medium $S$ is isotropic for the applied wavelengths, in addition to some further presuppositions developed throughout the paper. (Electromagnetic radiation, 2020; Purcell \& Morin , 2013).

\section{Explanation}

\subsection{Parameters of the environment}

The specificity of the propagation of radio waves in substances is determined by their electromagnetic characteristics: dielectric constant $\hat{\varepsilon}$ and magnetic permeability $\hat{\mu}$, which are complex quantities with real and imaginary parts for bi-complex media, (Кобак, 1975; Финкельштейн, 1983):

$$
\begin{gathered}
\hat{\varepsilon}=\varepsilon^{\prime}+j \varepsilon^{\prime \prime}=\varepsilon^{\prime}+j\left(\frac{4 \pi s_{\varepsilon}}{\omega}\right), \varepsilon^{\prime}=\varepsilon_{0} \varepsilon_{r} \\
\hat{\mu}=\mu^{\prime}+j \mu^{\prime \prime}=\mu^{\prime}+j\left(\frac{4 \pi s_{\mu}}{\omega}\right), \mu^{\prime}=\mu_{0} \mu_{r}
\end{gathered}
$$

Where $s_{\varepsilon}$, is a specific electrical (for a dielectrics or conductors) conductivity, and $s_{\mu}$ is a specific magnetic conductivity. The specific magnetic conductivity is called (permeance): $s_{\mu}=1 / \rho_{\mu}$, where $\rho_{\mu}$ (reluctance) is measured in $[1 / \mathrm{H}]$. In parentheses, we might say that the electrical conductivity of a biological substance is a variable and depends on the electrolyte balance of the organism, and changes during irradiation (Bioelectromagnetics, 2020). The process is not static and depends on the polarization of electrolytes in organisms when exposed to radio waves, and magnetic conductivity is physically associated with insignificant losses of magnetic energy in biological tissue and affects magnetic hysteresis, i.e. the medium is not linear, (Foster \& Schwan, 1995; Naydenov \& Yordanov, 2016). As proven, in a plane electromagnetic wave, the power loss for one period, averaged in unit volume, is $\left(s_{\varepsilon}+s_{\mu}\right) / 2$, (Вайнштейн, 1988). The refraction of the electromagnetic wave as a function of $\varepsilon$ and $\mu$ is also a complex quantity, as well as the complex wave resistance, important in the study of biological structures: 


$$
\begin{aligned}
\hat{n} & =\sqrt{|\hat{\mu}| \mid \hat{\varepsilon}} \mid e^{j 1 / 2\left(\Delta_{\mu}+\Delta_{\varepsilon}\right)} \\
\widehat{W} & =\sqrt{\frac{\hat{\mu}}{\hat{\varepsilon}}}=\sqrt{\frac{|\hat{\mu}|}{|\hat{\varepsilon}|}} e^{j 1 / 2\left(\Delta_{\mu}-\Delta_{\varepsilon}\right)}
\end{aligned}
$$
from:

Where $\Delta_{\mu}$ and $\Delta_{\varepsilon}$, are angles of magnetic and dielectric losses, respectively, and are obtained

$$
\begin{aligned}
\hat{\varepsilon} & =|\hat{\varepsilon}| e^{j \Delta_{\varepsilon}} \\
\hat{\mu} & =|\hat{\mu}| e^{j \Delta_{\mu}}
\end{aligned}
$$

If the refractive index is a complex number, $\hat{n}=n^{`}+j n^{\prime \prime}$, several other important parameters of the substance can be set:

- the complex wave number will be: $k \hat{n}=k^{\prime}+j \alpha$

- the attenuation coefficient in the substance: $\alpha=k n^{\prime \prime}$

- the speed of propagation of the field in the substance is: $c^{\prime}=\frac{c}{n^{\prime}}$

- the wavelength / after penetrating the environment $/: \lambda^{\prime}=\frac{\lambda}{n^{\prime}}=c^{\prime} / f$

- the wave number in the substance: $k^{\prime}=\frac{2 \pi}{\lambda^{\prime}}=\frac{\omega}{c^{\prime}}=k n^{\prime}$

The relations for a field in vacuum must also be taken into account: $k=\frac{2 \pi}{\lambda}=\frac{\omega}{c}$ wave number for a field in vacuum; $\lambda$ - wavelength, $\omega=2 \pi f ; c$ - velocity of the radio wave in vacuum; $\lambda$ - wavelength in vacuum.

\subsection{Wave characteristic}

What follows is an accurate description of the most important parameters that define the magnetic field. In a boundless isotropic medium without sources, without charges and currents, we describe a harmonic electromagnetic field with the homogeneous Helmholtz equation, the solution of which corresponds to a plane wave. A plane monochromatic wave with random elliptical polarization propagating along the $\mathrm{Z}$ axis is characterized by complex amplitudes whose vectors are electric and magnetic field strengths, respectively:

$$
\begin{aligned}
& \mathrm{E}=\xi E=\xi E_{0} j^{j k n z}=\xi E_{0} j^{j k^{\prime} z=\alpha z} \\
& H=[N \xi] H=[N \xi] e^{j k^{\prime} z=\alpha z} E_{0} / W
\end{aligned}
$$

Where $\xi$, the complex axis of wave polarization, $\mathrm{N}$ is a real value in the direction of propagation, the direction coincides with the $\boldsymbol{Z}$ axis as we assumed above; $E, H$ complex amplitudes; $E_{0}$, initial amplitude; $k^{\prime}, \lambda^{\prime}, \alpha$ values described above, $(6,8)$.

\subsection{Irradiation on a flat surface with a flat wave}

Proposed hereto is a comprehensive explanation of a phenomena occurrence between two environments. Medium $\boldsymbol{A}$ is an air layer of small thickness. We assume a close location of the emitter antenna /can be a small wide-band radar, whose antenna is located next to the reflective surface $0.1 \mathrm{~cm}$ $50 \mathrm{~cm}$, in order to use the results for analysis in bio-environments/, we will consider the surface perfectly smooth, (Shane, 1995; Israel, 2017). The dimensions of the irregularities must be much smaller than the wavelength (1\%) in the medium $\boldsymbol{S}$ described by formula (14) and (15):

Equations should be centered and e-marked with the number on the right-hand side.

$$
\begin{gathered}
\lambda=\frac{\lambda \sqrt{2}}{\sqrt{\sqrt{\left(\varepsilon^{\prime} \mu^{\prime}-\varepsilon^{\prime \prime} \mu^{\prime \prime}\right)^{2}+\left(\varepsilon^{\prime} \mu^{\prime \prime}+\varepsilon^{\prime \prime} \mu^{\prime}\right)^{2}}+\left(\varepsilon^{\prime} \mu^{\prime}-\varepsilon^{\prime \prime} \mu^{\prime \prime}\right)}} \\
\alpha=\frac{2 \pi}{\lambda^{\prime}}=\frac{\pi \sqrt{2} \sqrt{\sqrt{\left(\varepsilon^{\prime} \mu^{\prime}-\varepsilon^{\prime \prime} \mu^{\prime \prime}\right)^{2}+\left(\varepsilon^{\prime} \mu^{\prime \prime}+\varepsilon^{\prime \prime} \mu^{\prime}\right)^{2}}+\left(\varepsilon^{\prime} \mu^{\prime}-\varepsilon^{\prime \prime} \mu^{\prime \prime}\right)}}{\lambda}
\end{gathered}
$$


The attenuation and the wavelength in the medium are expressed by the parameters of the medium and $\gamma$ in the air falling on the plane. The inverse value of the attenuation is the depth of penetration. Medium $\boldsymbol{A}$ is air, medium $\boldsymbol{S}$ is an isotropic material with bi-complex parameters (above we assumed

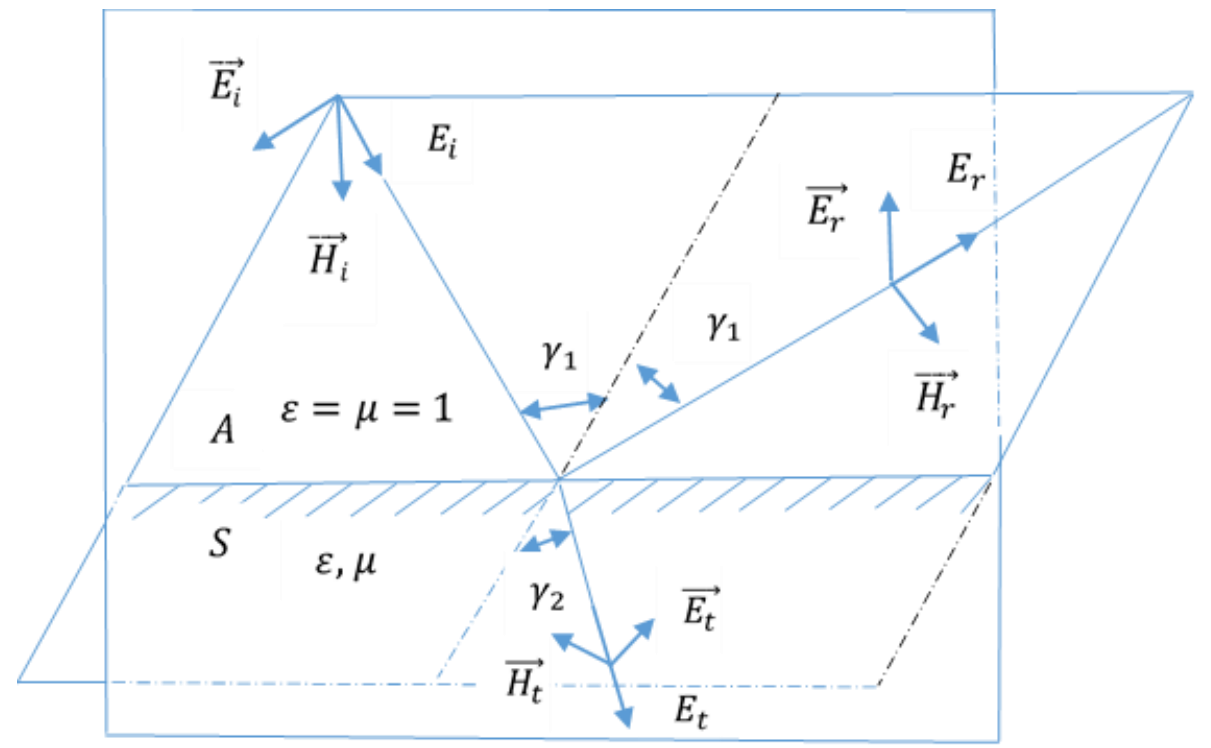

Fig. 1 Reflection and passage of a plane wave at the boundary of two environments.

that it can also be a bio substance), Fig. 1. We assume that a flat monochromatic wave with linear polarization and complex amplitude $E_{i}$ falls on the surface of the air-medium section at an angle $\gamma$. As a result of the interaction of the incident wave with the surface, a reflection $E_{r}$ occurs, propagating at an angle , mirrored along the normal and a refracted wave $E_{t}$ passing through the material (Snelius's law). The ratio of the amplitudes and phases of the incident and reflected waves are described by formulas for the complex coefficients of reflection and transmission (Kamburov, 2002; Kamburov \& Yordanov, 2018). Since a wave with random polarization decomposes into two waves with orthogonal linear polarizations, for the complete description of the surface properties it is enough to know the reflection and transition coefficients for both cases of linear polarization. $\mathbf{E}$ - polarization, when the vector $\mathbf{E}$ of the incident, reflected and a refracted wave lies in the plane of incidence and the vector $\mathbf{H}$ is perpendicular to it, and $\mathbf{H}$ - polarization when the vector $\mathbf{H}$ lies in the plane of incidence and the vector $\mathbf{E}$ is perpendicular to it. The coefficients of reflection and penetration corresponding to these cases, provided that the medium is a dielectric with negligible losses, we have:

$$
\begin{gathered}
R_{H}=\frac{\cos \gamma_{1}-\sqrt{\varepsilon^{\prime}-\sin ^{2} \gamma_{1}}}{\cos \gamma_{1}+\sqrt{\varepsilon^{\prime}-\sin ^{2} \gamma_{1}}}, R_{H}=\frac{\cos \gamma_{1}-\sqrt{\varepsilon^{\prime}-\sin ^{2} \gamma_{1}}}{\cos \gamma_{1}+\sqrt{\varepsilon^{\prime}-\sin ^{2} \gamma_{1}}}, \\
T_{E}=\frac{2 \sqrt{\varepsilon^{\prime}} \cos \gamma_{1}}{\sqrt{\varepsilon^{\prime}-\sin ^{2} \gamma_{1}}+\varepsilon^{\prime} \cos \gamma_{1}}, T_{H}=\frac{2 \cos \psi_{1}}{\cos \psi_{1}+\sqrt{\varepsilon^{\prime}-\sin ^{2} \psi_{1}}},
\end{gathered}
$$

At $\gamma_{1} \approx 0$, have the form below:

$$
T_{E}=T_{H}=\frac{2}{1+\sqrt{\varepsilon^{\prime}}}, R_{E}=R_{H}=\frac{1-\sqrt{\varepsilon^{\prime}}}{1+\sqrt{\varepsilon^{\prime}}}
$$

For $\gamma_{1} \approx \pi / 2$, they have the form respectively:

$$
R_{E} \approx \frac{1-\sqrt{\varepsilon^{\prime}} \cdot \cos \gamma_{1}}{1+\sqrt{\varepsilon^{\prime}} \cdot \cos \gamma_{1}}, R_{H} \approx \frac{\cos \gamma_{1}-\sqrt{\varepsilon^{\prime}}}{\cos \gamma_{1}+\sqrt{\varepsilon^{\prime}}}, T_{E} \approx \frac{2 \cos \gamma_{1}}{1+\sqrt{\varepsilon^{\prime}} \cos \gamma_{1}}, T_{H} \approx \frac{2 \cos \gamma_{1}}{\cos \gamma_{1}+\sqrt{\varepsilon^{\prime}}}
$$


Thus, , provided that the medium is a metal or alloy with very high conductivity, obtained are the corresponding reflection coefficients:

$$
R_{E}=\frac{1-\sqrt{\frac{4 \pi s}{\omega}} \cdot e^{j \pi / 4} \cos \gamma_{1}}{1+\sqrt{\frac{4 \pi s}{\omega}} \cdot e^{j \pi / 4} \cos \gamma_{1}}, R_{H}=\frac{\cos \gamma_{1}-\sqrt{\frac{4 \pi s}{\omega}} \cdot e^{j \pi / 4}}{\cos \gamma_{1}+\sqrt{\frac{4 \pi s}{\omega}} \cdot e^{j \pi / 4}},
$$

At $\gamma_{1} \approx 0$, have the form below:

$$
R_{E, H}=-\left(1-\frac{1}{\sqrt{\frac{2 \pi s}{\omega}}}\right)-j \frac{1}{\sqrt{\frac{2 \pi s}{\omega}}}
$$

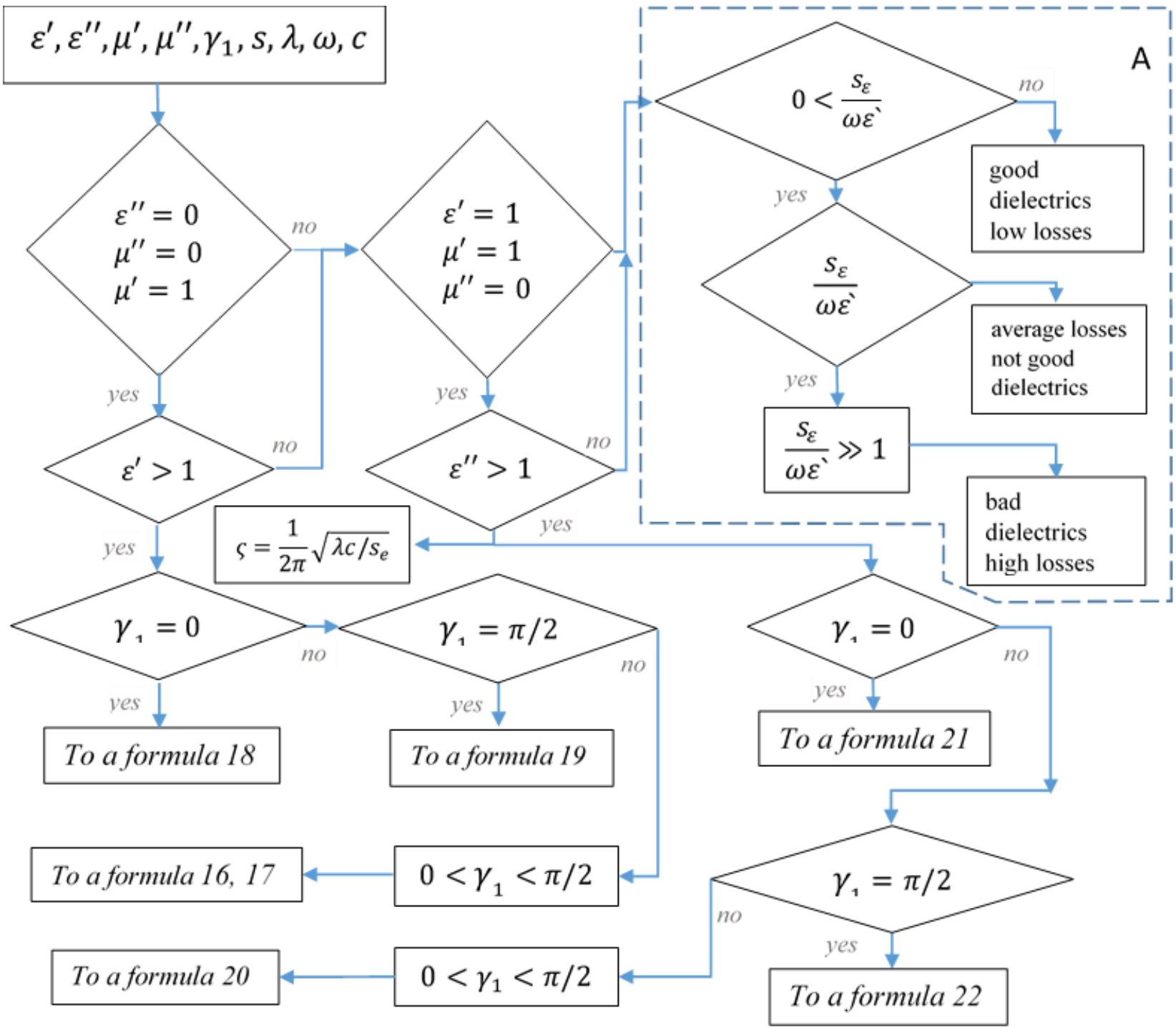

Fig. 2 Block diagram of an algorithm for determining the transmission and reflection coefficients $T$ and $\boldsymbol{R}$, respectively, depending on the angle of incidence $\gamma$, for a flat electromagnetic wave for the electric field strength for two limit states of $\varepsilon$ and $\mu$.

And for $\gamma_{1} \approx \pi / 2$, the reflection coefficients have the form respectively:

$$
R_{E}=-\frac{1-\frac{4 \pi \mathrm{s}}{\omega} \cos \gamma_{1}-j 2 \sqrt{\frac{2 \pi \mathrm{s}}{\omega}} \cos \gamma_{1}}{1+\frac{4 \pi \mathrm{s}}{\omega} \cos \gamma_{1}+j 2 \sqrt{\frac{2 \pi \mathrm{s}}{\omega}} c}, R_{H}=-1\left(1-\cos \gamma_{1}\right)-j \cos \gamma_{1}
$$


As illustrated, here the transition coefficients are not calculated, but only the penetration depth, which is a few microns for frequencies close to $1 \mathrm{GHz}$, to parts per micron for a range above $10 \mathrm{GHz}$.

\section{$3 \quad$ Block diagram of an algorithm}

For dielectric with very small losses or metal /alloy/ with very good conductivity, the limit values adopted are: $\varepsilon^{\prime \prime}=\mu^{\prime \prime} \rightarrow 0, \mu^{\prime}=1, \varepsilon=\varepsilon^{\prime}$ и $\varepsilon^{\prime}=\mu^{\prime} \rightarrow 1, \mu^{\prime \prime}=0, \varepsilon^{\prime \prime}=4 \pi s_{\varepsilon} / \omega$ respectively, in order to simplify the algorithm for establishing the reflection and transmission coefficients, only for such materials at ultra-high frequencies, when high accuracy is not required. Exact approximation to 0 and 1 is taken according to the type of substrate. Substances in which $\varepsilon^{\prime \prime} \neq 0, \mu^{\prime \prime} \neq 0, \varepsilon^{\prime} \neq 1$, $\mu^{\prime} \neq 1$ will be considered in another papers. In the enclosed field A, there may be bio-based substances, living matter, cellular structures, etc., fig. 2. Table 1 shows the depth of penetration of an electromagnetic field into a solid conductive medium with crystal structure metals, respectively alloys. Reported in Table 1 are the changes in the wavelengths of matter with the ratio $\frac{\lambda}{\lambda}$.

\section{Conclusion}

The algorithm is applicable for calculating the depth of penetration and the ratio of wavelengths external and internal to the environment in which the signal attenuates "e" times. Presented in Table. 1 are the values for some frequencies, by materials. The results are used in the design of microwave antennas and wideband microwave parametric filters.

Table 1. Depth of penetration $\zeta$ and $\lambda^{\wedge^{\prime} / \lambda}$

\begin{tabular}{|l|c|c|c|c|c|c|c|c|c|}
\hline \multirow{2}{*}{ Substance } & \multirow{2}{*}{$\mathrm{s}_{e}, \mathrm{~S} / \mathrm{m}$} & \multicolumn{2}{|c|}{$1 \mathrm{GHz}$} & \multicolumn{2}{c|}{$3 \mathrm{GHz}$} & \multicolumn{2}{c|}{$10 \mathrm{GHz}$} & \multicolumn{2}{c|}{$30 \mathrm{GHz}$} \\
\hline $\mathrm{Cu}$ & $5,96.10^{7}$ & $1,9.10^{-6}$ & $40.10^{-6}$ & $1,1.10^{-6}$ & $70.10^{-6}$ & $0,61.10^{-6}$ & $129.10^{-6}$ & $0,35.10^{-6}$ & $224.10^{-6}$ \\
\hline $\mathrm{Al}$ & $3,77.10^{7}$ & $2,5.10^{-6}$ & $51.10^{-6}$ & $1,4.10^{-6}$ & $89.10^{-6}$ & $0,77.10^{-6}$ & $162.10^{-6}$ & $0,45.10^{-6}$ & $282.10^{-6}$ \\
\hline $\mathrm{Fe}$ & $1.10^{7}$ & $4,8.10^{-6}$ & $100.10^{-6}$ & $2,7.10^{-6}$ & $173.10^{-6}$ & $1,5.10^{-6}$ & $316.10^{-6}$ & $0,87.10^{-6}$ & $547.10^{-6}$ \\
\hline
\end{tabular}

\section{References}

Purcell, E., \& Morin, D. (2013). Electricity and Magnetism (3rd ed.). Cambridge: Cambridge University Press. https://doi.org/10.1017/CBO9781139012973

Foster K.R. \& Schwan H. P. (1996) Dielectric properties of tissues. In: C. Polk, E. Postow (Eds.), Handbook of Biological Effects of Electromagnetic Fields (2 $\left.{ }^{\text {nd }} e d\right)$ (pp. 25-102), CRC Press.

Kamburov, L. \& Yordanov, A. (2018). Reflector antenna with discrete flat reflectors. Acoustics magazine, issue 20, 2018. The National Conference "Acoustics 2018", 10.2018, Varna, ISSN 13124897.

Israel M. (2017). Guide to protection against non-ionizing radiation. Non-ionizing radiation, man and the environment. National Center for Public Health and Analysis. ISBN 978-954-8404-35-8.

Naydenov, B. G., \& Yordanov, A. (2016). Analysis of the methods and techniques applied in radar sensors for contactless diagnostics based on EM reflection of the boundary between the two materials. Scientific conference with international participation MATTEX 2016, October 1113, 2016, Shumen. ISSN:1314-392. 
Kamburov, L. (2002) Fresnel-Zone Antennas with Dielectric Phase-Correcting Zones. XXXVII International Scientific Conference on Information, Communication and Energy Systems and Technologies. 1-4 OCTOBER 2002 Nish Yugoslavia, ISBN: 86-80135-69-0.

Shane, C. (1995). An Introduction to Electromagnetic Wave Propagation and Antennas. Springer Science and Business Media. ISBN 978-0387915012.

Вайнштейн, Л. А. (1988). Электромагнитные волны. Москва, Радио и Связь, ISBN 5-256-000640

Кобак В. О. (1975). Радиолокаџионнъйе отражатели. М.: Радио и Связь, UDC 621.396.96

Финкельштейн, М. И.(1983). Основъй радиолокащий. 2-ое изд. М.: Радио и Связь, UDC $621.396 .96(075)$

Electromagnetic radiation. (n.d.). Wikipedia. Retrieved October 22, 2020 from https://en.wikipedia.org/wiki/Electromagnetic_radiation

Bioelectromagnetics.(n.d.). (2020). Wikipedia. Retrieved $\quad$ October 22, 2020 from https://en.wikipedia.org/wiki/ Bioelectromagnetics 